\title{
Cancer Survivorship Research
}

National Cancer Institute

\section{Source}

National Cancer Institute. Cancer Survivorship Research. NCI Thesaurus. Code C18674.

Research on cancer survivors, covers the full spectrum of biomedical and psycho-social issues that arise at the point of discontinuation of cancer-specific therapy to the point of terminal diagnosis (6 months or less to live). 